\title{
RESPONSE MONITORING IN FOLLICULAR LYMPHOMA BY SYNCHRONOUS EVALUATION OF MINIMAL RESIDUAL DISEASE AND DIFFUSION-WEIGHTED MRI
}

\author{
A.N. Meleshko', S.A. Kharuzhyk ${ }^{2}$, N.A. Piatrouskaya, ${ }^{2, *}$ \\ ${ }^{1}$ Center for Pediatric Oncology, Hematology and Immunology, Minsk 223053, Belarus \\ ${ }^{2}$ N.N. Alexandrov National Cancer Center of Belarus, Minsk 223040, Belarus
}

\begin{abstract}
Aim: To demonstrate quantitative assessment of tumor response to treatment in patients with follicular lymphoma using parallel monitoring of minimal residual disease (MRD) and diffusion-weighted MRI (MRI-DWI) derived apparent diffusion coefficient (ADC). Materials and Methods: Two patients with follicular lymphoma were undergone synchronous evaluation of MRD and MRI-DWI at definite time points before, during and after chemotherapy. MRD level was calculated in diagnostic and follow up samples relative to the highest level of amplification of the target. Allele-specific primer for clonal IgH gene rearrangement was used as a target for real-time quantitative polymerase chain reaction (PCR). 1.5 Tesla scanner was used for MRI-DWI. The largest non necrotic lymph node was chosen for serial ADC measurement. Results: In first patient MRD reduced drastically in blood after chemotherapy but persisted at low level in bone marrow. Whole body MRI-DWI demonstrated regression of most of tumor lesions except one marginally enlarged iliac lymph node and allowed to predict tumor progression in this particular anatomical site based on low ADC value after treatment. In the second patient all three methods (MRD, ADC and radiologic evaluation) gave concordant result of complete tumor response and patient remained in a clinical remission during follow-up time. Conclusion: Quantitative PCR measurement may detect very low level of MRD in patients with follicular lymphoma which may have prognostic value. Combination of both PCR-based MRD and quantitative evaluation of MRI-DWI derived ADC provides safe added-value disease monitoring in lymphoma.
\end{abstract}

Key Words: follicular lymphoma, minimal residual disease, diffusion-weighed MRI, apparent diffusion coefficient, response monitoring.

Achievements in the field of non-Hodgkin lymphomas (NHL) molecular biology lead to progressive development of treatment personalization. At the same time they provide an option for accurate response monitoring and prognostication. A range of laboratory molecular techniques are available in lymphoma [1]. Evaluation of minimal residual disease (MRD) is used as supporting and additional data which help in response assessment fulfilled by conventional clinical and diagnostic imaging methods [2]. MRD is defined as any approach aimed at detecting and possibly quantifying residual tumor cells beyond the sensitivity level of routine imaging and laboratory techniques [3]. MRD has established role in leukaemias where it is used to detect persistent leukaemic cells in bone marrow or blood. High sensitivity of the test allows detecting leukaemic cells at level down to one cell per million. It was shown that MRD level after chemotherapy for acute lymphoblastic leukaemia is closely related to the probability of relapse [4]. To lesser extent MRD was studded in patients with indolent NHL, primarily follicular and mantle cell lymphomas [5-7].

Computed tomography (CT) or ${ }^{18} \mathrm{~F}$-fluorodeoxyglucose-PET are standard methods for diagnostics, response evaluation and follow-up of patients with

Submitted: December 01, 2015.

${ }^{*}$ Correspondence: E-mail: savitri@tut.by

Abbreviations used: ADC - apparent diffusion coefficient;

CT - computed tomography; DWI - diffusion-weighted imaging;

FDG - fluorodeoxyglucose; MRI - magnetic resonance imaging;

MRD - minimal residual disease; NHL - non-Hodgkin lymphoma;

PET - positron emission tomography; RQ-PCR - real-time quantitative polymerase chain reaction.
NHL. Before, during and after treatment numerous CT and FDG-PET exams are performed that leads to accumulation of ionizing radiation dose and increasing risk of secondary malignancy development, especially in children and young patients [8,9]. MRI with diffusion-weighed imaging (MRI-DWI) is a radiation-free and highly sensitive method for detection of nodal and extranodal lesions in lymphoma $[10,11]$. DWI is a MRI pulse sequence specially designed to detect microscopic water molecules movement in tissues and fluids in vivo. In solid tumors water diffusion takes place mainly in the intercellular spaces. In lymphomas diffusion in restricted because of increased cellular density and high nuclear to cytoplasm ratio allowing to characterize tumor at the cellular level. Because of high safety profile MRI-DWI may be repeated during and after treatment as many times as needed for treatment monitoring and follow-up. Another advantage of DWI compared to other MRI pulse sequences is a quantitative evaluation using apparent diffusion coefficient (ADC). Solid malignant tumors including lymphomas are characterized by low ADC, generally below $1-1.2 \cdot 10^{-3} \mathrm{~mm}^{2} / \mathrm{s}[12,13]$. During effective cytotoxic treatment ADC increases because of cell death mechanisms such as apoptosis and necrosis [14]. Due to quantitative nature MRD and MRI-DWI might be used in parallel to monitor tumor response in NHL.

\section{MATERIALS AND METHODS}

In frame of clinical projects "Idiotype DNA vaccines for B-cell NHL" and "MRI-DWI diagnostics in patients with lymphomas" synchronous evaluation of MRD and MRI-DWI derived ADC was performed in 2 patients with 
follicular lymphoma at the definite time points before, during and after treatment. Patients got standard diagnostic imaging tests for tumor staging, response evaluation and follow-up according to current practice in our institution.

MRD detection was performed as previously described [15]. DNA from primary removed neck lymph node was used for target identification and standard curve construction. Allele-specific primer for clonal $\mathrm{IgH}$ gene rearrangement was used as a target for realtime quantitative polymerase chain reaction (RQ-PCR) measurement [16]. MRD level was calculated in diagnostic and follow up samples relative to the highest level of amplification of the target.

1.5 Tesla scanner was used for MRI-DWI. Scanning technique was previously described [11]. Staging and treatment response assessment was based on whole body MRI-DWI. For serial ADC measurement the largest non necrotic lymph node (target lesion) was chosen on staging MRI-DWI. Lymph node was contoured on three diffusion-weighted images with largest tumor dimensions using scanner software. Contours were automatically transferred to corresponding ADC maps. Mean signal intensity on ADC map corresponds to tumor $A D C$ value. $A D C$ values on three slices were averaged in order to get a lesion ADC.

\section{RESULTS AND DISCUSSION}

A 28-year-old woman (patient 1) was diagnosed with follicular B-cell NHL involving axillary, mediastinal, mesenteric, paraaortic, iliac lymph nodes and bone marrow, Ann Arbor stage IV (Figure). Immunohistochemistry: $\mathrm{CD}_{20} \mathrm{O}^{++}, \mathrm{CD}^{-}, \mathrm{CD}^{-}$, cyclin D1- ${ }^{-}, \mathrm{CD}_{21}{ }^{-}, \mathrm{CD} 23^{-}, \mathrm{Ki}-67$ up to $20 \%$. A patient was undergone to eight courses of chemotherapy FC (fludarabine, cyclophosphamide). Before and after first, before and after second, before third course and after chemotherapy (8 courses) synchronous quantitative evaluation of MRD and ADC was performed
(Table 1). During chemotherapy PCR-based MRD reduced drastically in blood but persisted at low level in bone marrow. Paraaortic lymph node measuring $6.2 \times 4.2 \mathrm{~cm}$ was chosen for serial ADC evaluation. $A D C$ value of target lesion increased gradually from 0.51 to $1.59 \cdot 10^{-3} \mathrm{~mm}^{2} / \mathrm{s}$ corresponding to size normalization of most lymph nodes at restaging except right iliac one. ADC value of this lymph node was still low at the end of treatment $\left(0.95 \cdot 10^{-3} \mathrm{~mm}^{2} / \mathrm{s}\right)$ showing chemotherapy resistance. Tumor progression developed in this region one month later.

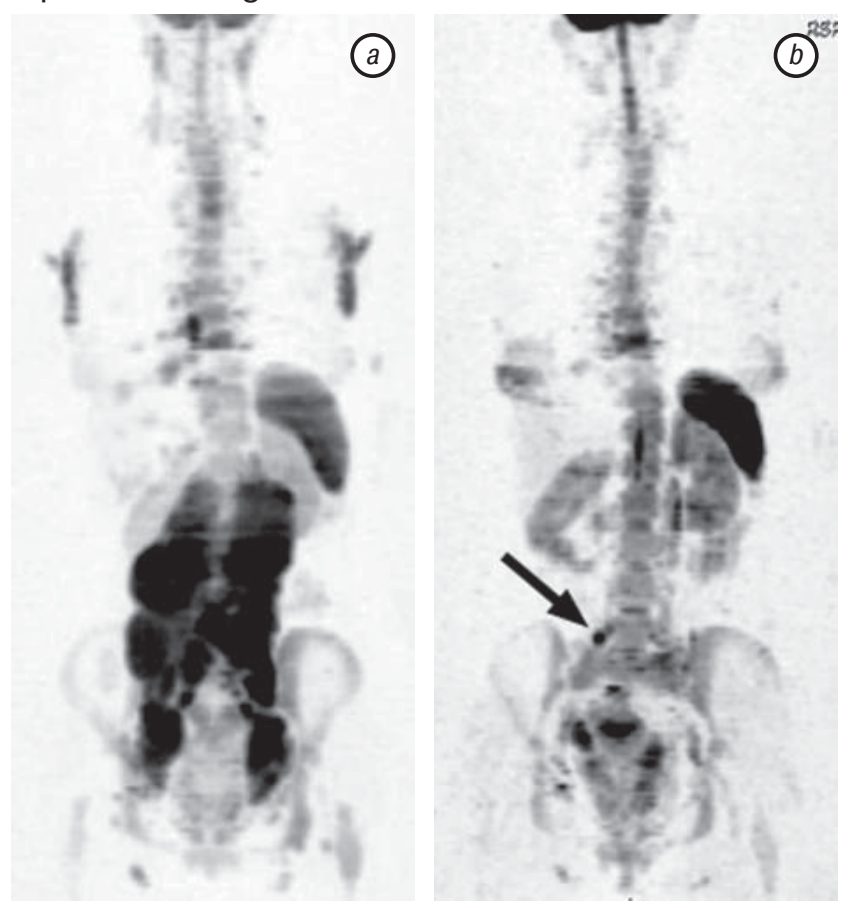

Figure. Whole body DWI of patient 1 with follicular lymphoma before treatment $(a)$ and after 8 courses of chemotherapy $(b)$. Inverted gray scale maximum intensity projection images are presented. Axillary, mediastinal, mesenteric, paraaortic and iliac lymph nodes were initially involved (a). After chemotherapy all lesions regressed except right iliac lymph node (arrow on $b$ ). Physiologic diffusion restriction in the brain, spinal cord, spleen, ovaries and endometrium is noted

Table 1. Serial MRD, ADC and radiological evaluation in patient 1 with follicular NHL

\begin{tabular}{|c|c|c|c|}
\hline Time point & MRD & $\mathrm{ADC}, \times 10^{-3} \mathrm{~mm}^{2} / \mathrm{s}$ & Radiologic evaluation \\
\hline 1 month before treatment & $\begin{array}{l}\text { Tissue of lymph node } 85.85 \% \\
\text { Peripheral blood } 2.52 \%\end{array}$ & - & - \\
\hline Staging immediately before treatment & $\begin{array}{l}\text { Peripheral blood } 15 \% \\
\text { Bone marrow } 100 \%\end{array}$ & $\begin{array}{l}\text { Paraaortic lymph node } 0.51 \\
\text { Right iliac lymph node } 0.59\end{array}$ & $\begin{array}{l}\text { Whole body MRI-DWI: lesions of left axillary, mediastinal, } \\
\text { mesenteric, paraaortic, iliac lymph nodes and bone marrow }\end{array}$ \\
\hline Immediately after FC 1 & - & Paraaortic lymph node 0.75 & - \\
\hline Immediately before FC 2 & Peripheral blood $0.002 \%$ & Paraaortic lymph node 0.96 & - \\
\hline Immediately after FC 2 & Peripheral blood $0.008 \%$ & Paraaortic lymph node 1.15 & - \\
\hline Immediately before FC 3 & Peripheral blood $0 \%$ & Paraaortic lymph node 1.19 & - \\
\hline $\begin{array}{l}\text { Restaging after } 8 \text { courses of che- } \\
\text { motherapy (partial response) }\end{array}$ & $\begin{array}{l}\text { Peripheral blood } 0 \% \\
\text { Bone marrow } 0.002 \%\end{array}$ & $\begin{array}{l}\text { Paraaortic lymph node } 1.59 \\
\text { Right iliac lymph node } 0.95\end{array}$ & $\begin{array}{l}\text { Whole body MRI-DWI: upper diaphragm all lesions } \\
\text { regressed, lower diaphragm - right iliac lymph } \\
\text { nodes up to } 1.3 \mathrm{~cm}\end{array}$ \\
\hline $\begin{array}{l}\text { Follow-up } 2 \text { months after treatment } \\
\text { (progression) }\end{array}$ & Peripheral blood $0.002 \%$ & - & Ultrasound: right iliac lymph node enlarged to $2.5 \times 1.6 \mathrm{~cm}$ \\
\hline $\begin{array}{l}4 \text { months follow-up (further pro- } \\
\text { gression) }\end{array}$ & Peripheral blood 0.009\% & - & $\begin{array}{l}\text { CT scan: right iliac lymph node enlarged to } 4.9 \times 4.5 \mathrm{~cm} \text {, } \\
\text { all other lymph nodes are normal in size }\end{array}$ \\
\hline
\end{tabular}

Table 2. Serial MRD, ADC and radiological evaluation in patient 2 with follicular $\mathrm{NHL}$

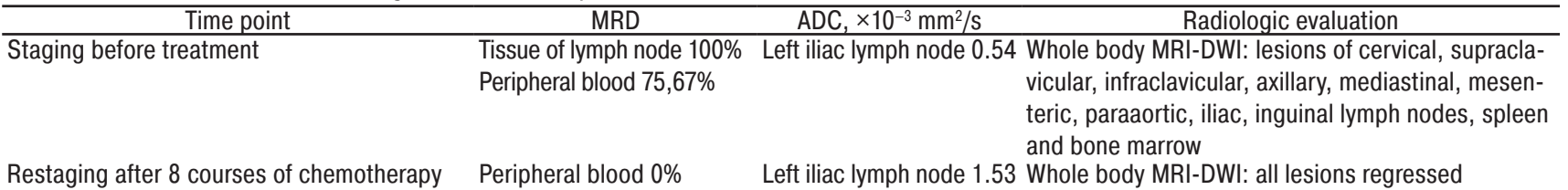


A patient 2 is a 43-year-old woman diagnosed with follicular B-cell NHL involving cervical, supraclavicular, infraclavicular, axillary, mediastinal, mesenteric, paraaortic, iliac, inguinal lymph nodes, spleen and bone marrow, Ann Arbor stage IV. Immunohistochemistry: $\mathrm{CD}_{2} \mathrm{O}^{++}, \mathrm{bcl6}^{+++}, \mathrm{CD} 10^{+++}, \mathrm{bcl} 2^{++}, \mathrm{CD} 21^{-/+}$, cyclin $\mathrm{D} 1^{-}$, $\mathrm{CD5}^{-}, \mathrm{CD}^{-}$, Ki-67 more than $40 \%$. A patient was undergone to eight courses of chemotherapy R-CHOP (rituximab, cyclophosphamide, doxorubicin, vincristine, prednisolon). Before and after chemotherapy (8 courses) synchronous quantitative evaluation of MRD and ADC was performed (Table 2). Left iliac lymph node was chosen as a target for ADC measurement. After end of chemotherapy MRD was not detectable in the blood any more, target lesion ADC increased to the level above $1.5 \cdot 10^{-3} \mathrm{~mm}^{2} / \mathrm{s}$ and radiological evaluation showed complete response of all tumors. Patient remains in remission during 6 months of follow-up.

In this report we present clinical cases of two patients with follicular NHL treated with chemotherapy with serial MRD and MRI-DWI examination. Results of MRD, ADC and radiological evaluation at different time points before, during and after treatment are presented. In patient 1 whole body MRI-DWI after eight courses of chemotherapy demonstrated regression of most of tumor lesions. Only iliac lymph node remained marginally enlarged measuring $1.3 \mathrm{~cm}$. Such small lymph nodes might be difficult to detect and even more difficult to characterize by conventional imaging techniques such as CT, ultrasonography or MRI. DWI with $A D C$ calculation is a functional imaging technique which allowed to predict tumor progression in this particular anatomical site based on low ADC value after treatment. In the patient 2 all three methods (MRD, $A D C$ and radiologic evaluation) gave concordant result of complete tumor response and patient remained in a clinical remission during follow-up time.

In the published literature MRD was used in patients with indolent lymphomas for molecular monitoring of treatment response and as a surrogate marker for prognosis. In follicular lymphoma negative PCR-based MRD status after rituximab treatment predicted higher freedom from recurrence rate $-57 \%$ compared with $20 \%$ in patients with molecular positivity [6]. In advance stage follicular lymphoma patients achieved an adequate remission with induction therapy PCR detectable disease in the bone marrow before autologous stem cell transplantation was the strongest predictor of decreased progression-free survival [17]. To best of our knowledge our report is the first one correlating MRD with relatively new quantitative functional imaging technique - diffusion-weighted MRI with ADC calculation.

In conclusion, quantitative PCR measurement may detect very low level of MRD in patients with follicular lymphoma which may have prognostic value. Combination of both PCR-based MRD and quantitative evaluation of MRI-DWI derived ADC provides added-value disease monitoring in lymphoma.

\section{REFERENCES}

1. Spagnolo DV, Ellis DW, Juneja S, et al. The role of molecular studies in lymphoma diagnosis: a review. Pathology 2004; 36: 19-44.

2. Cheson BD, Fisher RI, Barrington SF, et al. Recommendations for initial evaluation, staging, and response assessment of Hodgkin and non-Hodgkin lymphoma: the Lugano Classification. J Clin Oncol 2014; 32: 3059-68.

3. Lobetti-Bodoni C, Mantoan B, Monitillo L, et al. Clinical implications and prognostic role of minimal residual disease detection in follicular lymphoma. Ther Adv Hematol 2013; 4: 189-98.

4. Brisco MJ, Condon J, Hughes E, et al. Outcome prediction in childhood acute lymphoblastic leukaemia by molecular quantification of residual disease at the end of induction. Lancet 1994; 343: 196-200.

5. van der Velden VH, Hochhaus A, Cazzaniga G, et al. Detection of minimal residual disease in hematologic malignancies by real-time quantitative PCR: principles, approaches, and laboratory aspects. Leukemia 2003; 17: 1013-34.

6. Rambaldi A, Lazzari M, Carlotti E, et al. Monitoring of minimal residual disease after $\mathrm{CHOP}$ and rituximab in previously untreated patients with follicular lymphoma. Blood 2002; 99: 856-62.

7. Pott C. Minimal residual disease detection in mantle cell lymphoma: technical aspects and clinical relevance. Semin Hematol 2011; 48: 172-84.

8. Brenner DJ, Elliston CD. Estimated radiation risks potentially associated with full-body CT screening. Radiology 2004; 232: 735-38.

9. Kharuzhyk SA, Leusik EA. Repeated computed tomography examinations: radiation dose and radiation risk in malignant lymphomas. Radiat Biol Radioecol 2014; 54: 466-73 (in Russian).

10. Vermoolen MA, Kersten MJ, Fijnheer R, et al. Magnetic resonance imaging of malignant lymphoma. Exp Rev Hematol 2011; 4: 161-71.

11. Kharuzhyk SA, Zhavrid EA, Sachivko NV, et al. Possibilities of whole-body diffusion-weighted magnetic resonance imaging compared to X-ray computed tomography in staging lymphoma. Oncol J 2015; 9: 43-8 (in Russian).

12. Punwani S, Prakash V, Bainbridge A, et al. Quantitative diffusion weighted MRI: a functional biomarker of nodal disease in Hodgkin lymphoma? Cancer Biomark 2010; 7: 249-59.

13. Wu X, Pertovaara H, Dastidar P, et al. ADC measurements in diffuse large B-cell lymphoma and follicular lymphoma: a DWI and cellularity study. Eur J Radiol 2013; 82: e158-64.

14. Padhani AR, Liu G, Koh DM, et al. Diffusion-weighted magnetic resonance imaging as a cancer biomarker: consensus and recommendations. Neoplasia 2009; 11: 102-25.

15. Meleshko AN, Savva NN, Fedasenka UU, et al. Prognostic value of MRD-dynamics in childhood acute lymphoblastic leukemia treated according to the MB-2002/2008 protocols. Leuk Res 2011; 35: 1312-20.

16. Pott C, Brüggemann M, Ritgen M, et al. MRD detection in B-cell non-Hodgkin lymphomas using Ig gene rearrangements and chromosomal translocations as targets for realtime quantitative PCR. Methods Mol Biol 2013; 971: 175-200.

17. Brown JR, Feng Y, Gribben JG, et al. Long-term survival after autologous bone marrow transplantation for follicular lymphoma in first remission. Biol Blood Marrow Transplant 2007; 13: 1057-65.

Copyright @ Experimental Oncology, 2015 